\title{
Crescimento inicial de curauá e paricá submetidos à aplicação de extrato vegetal de
} curauá

\section{Initial growth of curauá and paricá under the application of curauá's vegetal extract}

\author{
Iracema Maria Cordeiro ${ }^{1}{ }^{1}$; Gustavo Schwartz ${ }^{\left(\mathbb{D}^{2}\right.}{ }^{2}$ Gracialda Costa Ferreira ${ }^{3}{ }^{3}$; Osmar Alves Lameira ${ }^{4}$
}

${ }^{1}$ Doutora em Ciências Florestais, Programa de Pós-Graduação em Ciências Florestais, Universidade Federal Rural da Amazônia, Belém, Pará, (91) 992071629, iracema3c@gmail.com; ²Doutor em Ecologia e Manejo Florestal, Embrapa Amazônia Oriental, Belém, Pará, (91) 3204-1000, gustavo.schwartz@embrapa.br; ${ }^{3}$ Doutora em Botânica, Universidade Federal Rural da Amazônia, Belém, Pará, (91) 992071629, gracialdaf@yahoo.com.br; ${ }^{4}$ Doutor em Agronomia, Embrapa Amazônia Oriental, Belém, Pará, (91) 3204-1000, osmar.lameira@embrapa.br

\section{N O T A}

C I E N T Í F I C A

Recebido: $29 / 04 / 2019$

Aprovado: 27/09/2019

\section{Palavras-chave:}

Biomassa

Adubo orgânico

Aproveitamento de resíduos

Key words:

Biomass

Organic fertilizer

Waste management

\section{R E S U M O}

Este estudo teve como objetivo testar o efeito da fertilização do solo com extrato vegetal de curauá (Ananas erectifolius) sobre o crescimento de indivíduos de curauá e paricá (Schizolobium parahyba var. amazonicum) plantados em consórcio. O trabalho foi conduzido no campo experimental da empresa Tramontina Belém, município de Aurora do Pará, PA, Brasil. O experimento seguiu um delineamento inteiramente casualizado, com cinco tratamentos e quatro repetições por tratamento, totalizando 20 parcelas experimentais. $\mathrm{O}$ espaçamento adotado foi de 4,0 m x 3,0 m entre indivíduos de paricá e 0,80 m x 0,80 m entre as plantas de curauá. Os cinco tratamentos consistiram em diferentes concentrações de extrato vegetal de curauá: $100 \%$ extrato; $75 \%$ extrato e $25 \%$ água; $50 \%$ extrato e $50 \%$ água; $25 \%$ extrato e $75 \%$ água e uma testemunha com $100 \%$ água. O extrato vegetal de curauá foi aplicado mensalmente por um período de seis meses e as variáveis analisadas foram: número e comprimento de folhas de curauá e altura e diâmetro de paricá. A partir dos resultados obtidos, pode-se observar que houve efeito positivo da fertilização do solo quando utilizou o extrato vegetal de curauá na concentração de $25 \%$ em relação ao número e comprimento de folhas de curauá, assim como também pode inferir que ocorreram resultado positivo na concentração de $75 \%$ para o crescimento em altura e diâmetro de paricá. O residuo de curauá pode ser usado como adubo orgânico em plantações consorciadas, no entanto estudos em mais detalhes são necessários para permitir indicações de concentrações mais eficientes para o crescimento das plantas de curauá e paricá.

\section{A B S T R A C T}

This study had the objective to test the effect of soil fertilization with curauá's vegetal extract (Ananas erectifolius) over the growth of individuals of curauá and paricá (Schizolobium parahyba var. amazonicum) planted in consortium. The work was carried out in the experimental field of the company Tramontina Belém, municipality of Aurora do Pará, PA, Brazil. The experiment followed a fully randomized experimental design, with five treatments and four repetitions per treatment, totaling 20 experimental plots. The adopted planting spacing was $4.0 \mathrm{~m}$ x $3.0 \mathrm{~m}$ among individuals of paricá and $0.80 \mathrm{~m} \times 0.80$ $\mathrm{m}$ among plants of curauá. The five treatments consisted in different concentrations of curauá's vegetal extract: $100 \%$ extract; $75 \%$ extract and 25\% water; (3) 50\% extract and $50 \%$ water; (4) $25 \%$ extract and $75 \%$ water and (5) a control with $100 \%$ water. The curauá's vegetal extract was applied in a monthly basis during six months and the assessed variables were: number and length of curauá's leaves and height and diameter of paricá. From the results obtained, it is possible to observe that there was a positive effect of soil fertilization when the curauá's vegetal extract was used in the concentration of $25 \%$ over the number and length of curauá's leaves, as well as it is possible to infer that positive results occurred in the concentration of $75 \%$ over growth in height and diameter of paricá. The curauá's vegetal extract can be used as organic fertilizer in consortium plantings, however studies in more detail are necessary to permit indications of more efficient concentrations for the growth of individuals of curauá and paricá.
Revista Verde

ISSN 1981-8203

Pombal, Paraíba, Brasil v. 14 , n.4, out.-dez, p.586-590, 2019

doi: $10.18378 /$ rvads.v14i4.6515 


\section{INTRODUÇÃO}

Nos países em desenvolvimento têm sido construídas muitas alternativas de uso da terra que atendam tanto a necessidades de produção como a princípios agroecológicos, o que vem impulsionando a demanda por sistemas de cultivo mais sustentáveis (CORDEIRO et al., 2009). Nesse sentido, os sistemas agroflorestais com base agroecológica aparecem como uma importante alternativa de preservação de florestas nativas e de uso racional do solo (JOSE, 2009).

$\mathrm{Na}$ Região Amazônica, dentre as espécies nativas potencias para plantios em sistemas agroflorestais encontramse o curauá (Ananas. erectifolius (L.B.Sm.) Coppens \& F.Leal - Bromeliaceae) e o paricá (Schizolobium parahyba var. amazonicum (Huber ex Ducke) Barneby - Fabaceae) (CORDEIRO et al., 2016). De modo que, o cultivo com as espécies citadas vem assumindo papel de destaque na economia do estado do Pará, não somente por apresentarem diversos usos e boa adaptabilidade a diferentes condições ambientais, mas por criar oportunidade de emprego e renda com benefícios socioeconômicos e ecológicos (CORDEIRO et al., 2016).

O curauá é uma planta fibrosa, monocotiledônea, herbácea e pouco exigente quanto à fertilidade do solo (LEDO, 1967). Seu principal produto, a fibra, de excelente qualidade e resistência, é utilizado na fabricação de papel, confecção de cordas, barbantes, produtos artesanais, na indústria geotêxtil e automobilística para produção de componentes para bancos e revestimento de automóveis (MONTEIRO et al., 2010). Além da fibra, as folhas do curauá produzem durante o processo de desfibramento os subprodutos (resíduos) como: mucilagem e extrato vegetal (soro). Esses resíduos juntos respondem por $90 \%$ de tudo o que é produzido no processamento, porém essa matéria prima é abandonada no campo, pois ainda não se dispõem de uma destinação econômica (CORDEIRO et al., 2016).

Cabe destacar que, o extrato vegetal de curauá apresenta propriedades que potencializam o seu uso como um herbicida natural. Além disso, a mucilagem do curauá pode ser utilizada na fabricação de papel e como adubo orgânico. Partindo dessa perspectiva, o uso de adubos orgânicos ou adubos verdes na agricultura atualmente tem sido recomendada para manter ou incrementar os teores de matéria orgânica no solo (FERNANDES et al., 1998), bem como inibir o crescimento de ervas daninhas, o que pode levar a um aumento na eficiência de utilização da água e dos nutrientes pelas plantas (RAVEN et al., 2001).

O paricá é uma árvore nativa da região Amazônica, podendo ser encontrada em floresta primária e secundária, tanto em solos de terra firme como em várzea alta (DUCKE, 1939). Esta pode alcançar altura de até $30 \mathrm{~m}$, sendo razoavelmente imune ao ataque de pragas e doenças (CORDEIRO et al., 2015). A madeira do paricá tem sido usada para a fabricação de laminados e compensados e, além disso, é indicada para o emprego em vigas de madeira laminada colada-MLC (TEREZO e SZÜCS, 2010). Por possuir rápido crescimento e boa adaptabilidade, a espécie vem sendo bastante cultivada em plantações homogêneas, consórcios e em sistemas agroflorestais (RUIVO et al., 2007; CORDEIRO et al., 2009). Esta espécie também pode ser utilizada como uma alternativa de uso para recuperação de florestas alteradas e/ou degradadas na região amazônica (SCHWARTZ et al., 2017).

Estudos desenvolvidos em um sistema de cultivo com as espécies curauá e paricá, Cordeiro et al. (2009) observaram que os indivíduos de ambas as espécies são mutuamente beneficiados, de forma quantitativa e qualitativa, demonstrando haver interação positiva entre as especies.

Nesse contexto, informações sobre o desenvolvimento do consórcio curauá-paricá assim como a sua capacidade de reabilitar a fertilidade do solo são de grande relevância para o desenvolvimento de sistemas agroflorestais com bases agroecológicas. Desta forma, o objetivo da presente pesquisa foi avaliar o efeito de diferentes concentrações de extrato vegetal de curauá no crescimento de indivíduos de curauá e paricá em consórcio.

\section{MATERIAL E MÉTODOS}

O experimento foi implantado em janeiro de 2009 no campo experimental da empresa Tramontina Belém,

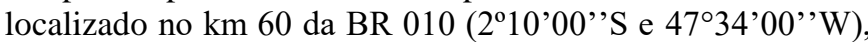
no município de Aurora do Pará, mesorregião do Nordeste Paraense, Pará, Brasil, a 210 km da capital do estado, Belém. Este foi realizado em parceria com a empresa Tramontina Belém, Embrapa Amazônia Oriental e Universidade Federal Rural da Amazônia no projeto "Potencialidade e uso de curauá em plantio florestal", financiado pela Fundação de Amparo a Pesquisa do Estado do Pará (FAPESPA).

O clima da área de estudo é do tipo Am3 pela classificação de Köppen (ANDRADE et al., 2017), com estação chuvosa entre os meses de janeiro a junho. A partir de dados obtidos pela estação meteorológica localizada a 2.000 $\mathrm{m}$ do experimento, obteve-se as seguintes informações para o intervalo de 2000 a 2014: a) precipitação $=2.900 \mathrm{~mm}$, b) temperatura $=26$ a $35^{\circ} \mathrm{C}$ e c) umidade média do ar $=74 \%$. Esta apresenta relevo de plano a suavemente ondulado. $\mathrm{O}$ solo da área é do tipo Latossolo Amarelo, de textura arenoargiloso, ácido, com baixa fertilidade química, porém, com boas propriedades físicas e média concentração de matéria orgânica (CORDEIRO, 2007; TRINDADE et al., 2009). Para melhor caracterização do solo, foi realizada uma análise na ocasião da instalação do experimento, cujos resultados mostraram um solo franco-arenoso levemente ácido $(\mathrm{pH}=$ $6,20$ ) e baixo $\mathrm{N}$, solo arenoso ácido ( $\mathrm{pH} 5,0)$ com valores de $\mathrm{N}=0,22 \mathrm{mg} / \mathrm{dm}^{3}$ e valores de $\mathrm{P}, \mathrm{K}, \mathrm{Ca}$ e $\mathrm{Mg}$ abaixo do nível crítico $\left(\mathrm{P}=1 \mathrm{mg} / \mathrm{dm}^{3}\right.$ e $\left.\mathrm{K}=31 \mathrm{mg} / \mathrm{dm}^{3}\right)$ segundo o Centro Internacional de Agricultura Tropical-CIAT (2010). Os valores das frações granulométricas $(\mathrm{g} / \mathrm{kg})$ de areia grossa $(138,75)$, areia fina $(552,5)$, silte (142) e argila total (190) na camada de $0-20 \mathrm{~cm}$ de profundidade também foram considerados baixos pelos critérios indicados por Vieira e Santos (1987) e Embrapa (1999).

O extrato de curauá foi obtido com o desfibramento das folhas com auxílio de uma máquina desfibradora volante do tipo embrap com duas lâminas. Vale ressaltar que, em média, a cada $100 \mathrm{~kg}$ de folhas desfibradas são gerados 26 litros de extrato. Em seguida foi acondicionado em recipientes plásticos e diluído em água constituindo os tratamentos nas seguintes concentrações: (1) $100 \%$ extrato; (2) $75 \%$ extrato e $25 \%$ água; (3) $50 \%$ extrato e $50 \%$ água; (4) $25 \%$ extrato e $75 \%$ água e (5) uma testemunha com $100 \%$ água. O extrato vegetal de curauá foi aplicado uma vez ao mês, por um 
período de seis meses. As variáveis levantadas foram número e comprimento de folhas de curauá e altura e diâmetro de paricá.

O experimento foi implantado em delineamento inteiramente casualizado com cinco tratamentos e quatro repetições cada, perfazendo um total de 20 parcelas experimentais. Mudas de paricá e curauá foram plantadas em consórcio. As mudas de paricá tinham em média $30 \mathrm{~cm}$ de altura e foram plantadas em espaçamento de 4,0 m x 3,0 m. Os indivíduos plantados de curauá apresentavam folhas com comprimento médio de $10 \mathrm{~cm}$, os quais foram plantados em espaçamento de $0,80 \mathrm{~m} \times 0,80 \mathrm{~m}$, onde se avaliou número e comprimento de folhas, enquanto que no paricá avaliou-se altura e diâmetro a 1,30 m do solo (DAP), nos intervalos de 60 e 180 dias. Os contrastes entre os tratamentos empregados foram comparados por meio de um modelo linear geral (GLM), e quando significante na ANOVA $(\mathrm{P}<0,05)$ analisado pelo teste de comparação de médias de Scott-Knott $(\mathrm{P}<0,05)$. Os valores médios dos tratamentos foram aplicados a modelos polinomiais de natureza quadrática. As funções polinomiais obtidas foram diferenciadas para obter os pontos de maximização das funções. As análises foram conduzidas com auxílio da planilha eletrônica Excel e com o pacote estatístico SAS System 5.6.

\section{RESULTADOS E DISCUSSÃO}

\section{Crescimento do curauá}

Houve efeito positivo para a concentração do extrato sobre o número e comprimento das folhas aos 60 e 180 dias, porem sem haver diferenças significativas a $95 \%$ de probabilidade (Tabela 1). Aos 60 dias após a implantação do experimento as plantas submetidas à aplicação das concentrações do extrato de curauá não apresentaram diferenças significativas, porém, se comparadas numericamente, plantas cultivadas nas concentrações de $25 \%$ e $50 \%$ apresentaram maior número de folhas, do que aquelas na concentração de $75 \%$ e de $100 \%$.

Tabela 1. Número de folhas de curauá aos 60 e 180 dias após o plantio e incremento mensal do número de folhas em função das concentrações de extrato vegetal de curauá utilizadas na fertilização do solo.

\begin{tabular}{cccc}
\hline & \multicolumn{2}{c}{ Número de folhas } & Incremento \\
\cline { 2 - 4 } Extrato vegetal $^{1}(\%)$ & 60 dias & 180 dias & Mensal \\
\hline $0^{2}$ & $14,11 \pm 5,47 \mathrm{~b}$ & $17,87 \pm 6,40 \mathrm{~b}$ & $1,88 \pm 3,81 \mathrm{~b}$ \\
25 & $14,91 \pm 4,64 \mathrm{ab}$ & $23,95 \pm 6,69 \mathrm{a}$ & $4,52 \pm 3,53 \mathrm{a}$ \\
70 & $17,50 \pm 5,78 \mathrm{a}$ & $24,29 \pm 5,51 \mathrm{a}$ & $3,40 \pm 4,05 \mathrm{ab}$ \\
100 & $16,63 \pm 5,07 \mathrm{ab}$ & $22,81 \pm 6,16 \mathrm{a}$ & $3,09 \pm 4,02 \mathrm{ab}$ \\
CV $(\%)$ & $15,27 \pm 6,07 \mathrm{ab}$ & $18,98 \pm 5,89 \mathrm{~b}$ & $1,86 \pm 4,88 \mathrm{~b}$ \\
\hline
\end{tabular}

${ }^{\mathrm{T}}$ Médias seguidas pela mesma letra na coluna, para cada concentração e tempo, não diferem entre si pelo modelo linear geral (GLM), ANOVA pelo teste ScottKnott $(\mathrm{P}<0,05) .{ }^{2} \mathrm{~A}$ concentração zero se refere à testemunha água. CV: Coeficiente de Variação geral.

Aos 180 dias os resultados sugerem que a aplicação do extrato vegetal até a concentração de $75 \%$ age positivamente no número de folhas (Tabela 1). Aos 16 meses, devido à influencia positiva no número e comprimento das folhas de curauá, a concentração de $75 \%$ extrato vegetal é indicada como adubação orgânica. $\mathrm{O}$ extrato vegetal colabora significativamente no crescimento das folhas da espécie, com a aplicação do extrato de curauá nas concentrações de $25 \%$ e $50 \%$.

Após a aplicação do ajuste polinomial, de natureza quadrática, foi observado que em todos os casos o modelo quadrático apresentou aderência. De modo que, por meio da diferenciação, foi possível estabelecer pontos de máximo aos $60 \operatorname{dias}(\mathrm{x}=59,05 \% ; \mathrm{y}=16,87$ folhas $)$, aos 180 dias $(\mathrm{x}=$
$50,85 \%$; y $=24,67$ folhas) e de incremento mensal de folhas ( $\mathrm{x}=46,25 \% ; \mathrm{y}=3,95$ folhas $/$ mês). Os resultados mostraram que os limiares ótimos estão entre 50 e $60 \%$ na concentração de extrato. Com esse indicador é possível optar pelo valor cumulativo aos 180 dias e recomendar a aplicação de 50\% de concentração do extrato para a obtenção maximizada de crescimento das folhas de curauá.

Em relação ao comprimento de folhas de curauá os valores encontrados apresentaram diferenças, tanto 60 dias quanto em 180 dias. Aos 60 dias, indivíduos cultivados com a aplicação do extrato vegetal na concentração de $25 \%$ diferiram estatisticamente $(\mathrm{P}<0,05)$ daqueles cultivados na concentração de $100 \%$ e da testemunha, porém não diferindo das demais concentrações (Tabela 2)

Tabela 2. Comprimento de folhas curauá aos 60 e 180 dias após o plantio e incremento mensal do comprimento das folhas em função das concentrações de extrato vegetal de curauá usadas na fertilização do solo.

\begin{tabular}{|c|c|c|c|}
\hline Tratamentos $^{1}$ & & $(\mathrm{~cm})$ & Incremento \\
\hline \multirow{2}{*}{$\begin{array}{l}\text { Conc de extrato } \\
\text { de curauá }(\%)\end{array}$} & & 180 & Mensal \\
\hline & \multicolumn{2}{|c|}{ Dias } & \\
\hline $0^{2}$ & $56,65 \pm 13,68 b$ & $103,27 \pm 19,51 \mathrm{a}$ & $23,31 \pm 11,69 a$ \\
\hline 25 & $64,02 \pm 18,86 a$ & $108,24 \pm 21,25 \mathrm{ab}$ & $22,11 \pm 13,20 \mathrm{a}$ \\
\hline 50 & $61,25 \pm 12,35 \mathrm{ab}$ & $114,32 \pm 14,9 \mathrm{a}$ & $26,54 \pm 8,51 \mathrm{a}$ \\
\hline 75 & $58,35 \pm 11,26 \mathrm{ab}$ & $108,17 \pm 16,98 \mathrm{ab}$ & $24,82 \pm 8,26 a$ \\
\hline 100 & $55,68 \pm 16,62 b$ & $101,70 \pm 22,56 b$ & $23,01 \pm 15,98 \mathrm{a}$ \\
\hline $\mathrm{CV}(\%)$ & 25,39 & 18,31 & 49,67 \\
\hline
\end{tabular}

${ }^{1}$ Médias seguidas pela mesma letra na coluna, para cada concentração e tempo, não diferem entre si pelo modelo linear geral (GLM), ANOVA pelo teste Scott-

Knott $(\mathrm{P}<0,05) .{ }^{2} \mathrm{~A}$ concentração zero se refere à testemunha água. CV: Coeficiente de Variação geral. 
Prabhu et al. (2010), empregando três diferentes substâncias (Panchakavya, ácido húmico e produtos orgânicos) com diferentes combinações de concentração encontraram que $2 \%$ Panchakavya $+0,2 \%$ de ácido húmico + $2 \%$ de extrato de folhas de moringa aos 30 e 60 dias após o plantio influenciou positivamente no crescimento e produção de manjericão sagrado (Ocimum sanctum).

O tratamento com concentração de $25 \%$ de extrato vegetal de curauá teve um efeito estatisticamente significativo no incremento mensal do número de folhas de curauá (Tabela 2). Um ajuste polinomial quadrático foi aplicado e, em todos os casos, a exceção do incremento mensal do comprimento de folhas, o modelo mostrou a melhor aderência. Através da diferenciação, foi possível estabelecer pontos de máximo, aos $60 \operatorname{dias}(\mathrm{x}=43,45 \% ; \mathrm{y}=62,16 \mathrm{~cm})$ e aos $180 \operatorname{dias}(\mathrm{x}=$ $48,45 \% ; \mathrm{y}=112,20 \mathrm{~cm})$. Considerando os valores de maximização da função quadrática, a concentração de $50 \%$ de extrato de curauá aparece como a melhor aplicação de adubo orgânico.

\section{Crescimento do paricá}

Não houve efeito estatisticamente significativo dos diferentes tratamentos de extrato de curauá sobre o crescimento em altura e DAP do paricá (Tabela 3).

Tabela 3. DAP e altura aos 60 e 180 dias após o plantio e incremento diário de altura e DAP de paricá em função das concentrações de extrato vegetal de curauá usadas na fertilização do solo.

\begin{tabular}{clllllr}
\hline \multirow{2}{*}{$\begin{array}{c}\text { Extrato Vegetal } \\
{ }^{1}\end{array}$} & \multicolumn{2}{c}{60 dias } & \multicolumn{2}{c}{180 dias } & \multicolumn{2}{c}{ Incremento Diário } \\
\cline { 2 - 7 } & DAP & $\begin{array}{l}\text { Alt } \\
(\mathrm{cm})\end{array}$ & $\begin{array}{l}\text { DAP } \\
(\mathrm{cm})\end{array}$ & $\begin{array}{l}\text { Alt } \\
(\mathrm{m})\end{array}$ & $\begin{array}{c}\text { DAP } \\
(\mathrm{cm})\end{array}$ & $\begin{array}{c}\text { Alt } \\
(\mathrm{m})\end{array}$ \\
\hline 0 & $15,58 \pm 2,14$ & $2,88 \pm 0,52$ & $32,14 \pm 4,33$ & $8,75 \pm 1,24$ & $0,24 \pm 0,15$ & $0,09 \pm 0,04$ \\
25 & $14,62 \pm 3,33$ & $2,54 \pm 0,77$ & $29,94 \pm 6,30$ & $8,50 \pm 1,80$ & $0,26 \pm 0,14$ & $0,10 \pm 0,04$ \\
50 & $15,68 \pm 2,90$ & $2,77 \pm 0,59$ & $31,47 \pm 3,86$ & $8,75 \pm 1,28$ & $0,26 \pm 0,09$ & $0,10 \pm 0,02$ \\
75 & $18,85 \pm 3,76$ & $3,21 \pm 0,72$ & $33,00 \pm 4,94$ & $10,22 \pm 1,80$ & $0,24 \pm 0,09$ & $0,12 \pm 0,04$ \\
100 & $14,60 \pm 2,68$ & $2,59 \pm 0,72$ & $27,94 \pm 4,28$ & $8,03 \pm 1,72$ & $0,22 \pm 0,10$ & $0,09 \pm 0,04$ \\
\hline CV $(\%)$ & 21,05 & 25,00 & 16,33 & 19,43 & 45,83 & 40,00 \\
\hline
\end{tabular}

DAP: diametro a altura do peito; Alt: altura; ${ }^{1}$ Médias seguidas pela mesma letra na coluna, para cada concentração e tempo, não diferem entre si pelo modelo linear geral (GLM), ANOVA pelo teste Scott-Knott $(\mathrm{P}<0,05) .{ }^{2}$ A concentração zero se refere à testemunha água. CV: Coeficiente de Variação geral.

O crescimento dos indivíduos de paricá pode ser explicado pelo papel que o extrato vegetal desempenha no solo, favorecendo o incremento da matéria orgânica e, consequentemente, melhor liberação de nutrientes essenciais para a cultura. Nesse sentido Ruivo et al., (2007) observaram que o paricá, quando cultivado em solo submetido à aplicação de adubação orgânica, as árvores apresentam maior diâmetro. Do mesmo modo Monteiro et al. (2010) estudando o crescimento de paricá com e sem adição de resíduos de madeira verificaram que as árvores que receberam cobertura no solo mostraram os maiores valores em DAP, entre 15 e 27 $\mathrm{cm}$.

A produtividade das espécies arbóreas com alto potencial de crescimento como o paricá é frequentemente limitada pela baixa fertilidade do solo e restrições nutricionais e hídricas. Assim, pelos resultados obtidos, a adição de extrato vegetal de curauá pode complementar os nutrientes necessários a indivíduos de paricá para maior crescimento e rendimento. No entanto estudos de mais longo prazo se fazem necessários.

\section{CONCLUSÕES}

O extrato vegetal do curauá nas concentração de $25 \%$ e $75 \%$ pode ser utilizado como adubo orgânico no cultivo de paricá, pois beneficia o crescimento das plantas consorciadas.

\section{AGRADECIMENTOS}

Os autores agradecem à empresa Tramontina Belém por disponibilizar a área de estudo e à Fundação de Aparo a Pesquisa do Estado do Pará - FAPESPA pelo financiamento do projeto PPAPE-017/2008.

\section{REFERÊNCIAS}

ANDRADE, V. M. S. de; CORDEIRO, I. M. C. C.; SCHWARTZ, G.; RANGEL-VASCONCELOS, L. G. T.;
OLIVEIRA, F. de A. Considerações sobre o clima e aspectos edafoclimáticos da mesorregião nordeste paraense. In: CORDEIRO, I. M. C. C.; RANGEL-VASCONCELOS, L. G. T.; SCHWARTZ, G.; OLIVEIRA, F. de A. (eds.) Nordeste Paraense: Panorama Geral e Uso Sustentável das Florestas Secundárias. Belém: EDUFRA, 2017. p.59-86.

\section{CIAT (CENTRO INTERNACIONAL DE AGRICULTURA}

TROPICAL). Restoring Amazonia: promoting Eco-Efficient land use alternatives $=$ Restauración de la Amazonia: fomento de alternativas Eco-Eficientes para uso del suelo. Turrialba, 2016.

CORDEIRO, I. M. C. C.; SANTANA, A. C.; LAMEIRA, O. A.; SILVA, I. M. Análise econômica dos sistemas de cultivo com Schizolobium parahyba var. amazonicum (Huber ex Ducke) Barneby (Paricá) e Ananas comosus var. erectifolius (L. B. Smith) Coppus \& Leal (Curauá) no município de Aurora do Pará (Pa), Brasil. Revista da Faculdade de Agronomia, v. 26, n.2, p. 243-265, 2009.

CORDEIRO, I. M. C. C.; OLIVEIRA JR. M. C.; GAZEL FILHO A. B.; BARROS, P. L. C. de; LAMEIRA, O. A.; OLIVEIRA F. DE A. Crecimiento del Schizolobium parahyba var. amazonicum cultivado en presencia de Ananas comosus var. erectifolius en Pará, Brasil. Agrociencia v.50, n.1, p.7988,2016

CORDEIRO I. M. C. C.; BARROS, P. L.C. de; LAMEIRA O.A.; GAZEL FILHO, A. B. Avaliação de plantios de paricá (Schizolobium parahyba var. amazonicum (huber ex Ducke) Barneby de diferentes idades e sistemas de cultivo no município de Aurora do Pará - Pa (Brasil). Ciência Florestal, v. 25 , n.3. p.679-687, 2015. 10.5902/1980509819618

CORDEIRO, I. M. C. C.; LAMEIRA O. A.; BARROS P. L. C. DE; MALHEIROS, M. A. da M. Comportamento do 
curauá sob diferentes níveis de radiação fotossinteticamente ativa em condições de cultivo. Revista Brasileira de Ciências Agrárias, v.5, n.1, p.49-53, 2010. 10.5039/agraria.v5i1a609

DUCKE, A. As Leguminosas da Amazônia Brasileira. Serviço florestal, Ministério da Agricultura, Serviço de Publicidade Agrícola. Rio de Janeiro, 1939. 88p.

EMBRAPA (CENTRO NACIONAL DE PESQUISA DE SOLOS). Sistema Brasileiro de Classificação de Solos. Embrapa Solos, Embrapa Produção de Informação, Rio de Janeiro, 412p, 1999.

FERNANDES, M. F.; BARRETO, A. C.; FILHO, J. E. Densidade de semeadura a lanço de sete leguminosas utilizadas como adubo verde em solos de tabuleiros costeiros. Embrapa-CPATC (Comunicado técnico), Aracaju, 1998.

JOSE, S. Agroforestry for ecosystem services and environmental benefits: An overview. Agroforestry Systems, v.76, n.1, p.1-10, 2009. 10.1007/s10457-009-9229-7

LEDO, I. A. DE M. O cultivo do curauá no lago grande de Franca. Belém: Banco da Amazônia S/A, 1967. 23 p.

MONTEIRO, K. F. G.; KERN, D. C.; RUIVO, M. DE L. P.; RODRIGUES, T. E; COMETTI, J. L. S. Uso de resíduos de madeira como alternativa de melhorar as condições ambientais em sistema de reflorestamento. Acta Amazonica, v.40, n.3, p. 409-414, 2010. 10.1590/S0044$\underline{59672010000300001}$

PRABHU, M.; KUMAR, A.; RAJAMANI, K. Influence of different organic substances on growth and herb yield of sacred basil (Ocimum sanctum L.). Indian Journal of Agricultural Research, v.44, n.1, p.48-52, 2010.

RAVEN, P. H.; EVERT, R. F.; EICHHORN, S. E. Biologia vegetal. Rio de Janeiro: Guanabara Koogan, 2001. 906p.

RUIVO, M. DE L. P.; MONTEIRO, K. F. G.; SILVA R. M. DA; SILVEIRA, I. M. DA, QUARESMA, H. D. DE A. B.; SÁ, L. D. A.; PROST, M. T. R. DA C. Gestão florestal e implicações sócio-ambientais na Amazônia Oriental. Oecologia Brasiliensis, v.11, n.4, p.481-492, 2007. $\underline{10.4257 / \mathrm{oeco} .2007 .1104 .02}$

SCHWARTZ, G.; PEREIRA, P. C. G.; SIVIERO, M. A.; PEREIRA, J. F.; RUSCHEL, A. R.; YARED, J. A. G. Enrichment planting in logging gaps with Schizolobium parahyba var. amazonicum (Huber ex Ducke) Barneby: A financially profitable alternative for degraded tropical forests in the Amazon. Forest Ecology and Management, v.390, p. 166-172, 2017. 10.1016/j.foreco.2017.01.031

TEREZO, R. F; SZÜCS, C. A. Análise de desempenho de vigas em madeira laminada colada de paricá (Schizolobium amazonicum Huber ex. Ducke). Scientia Forestalis, v.38, n.87, p.471-480, 2010.

TRINDADE, E. F. DA S.; RODRIGUES, T. E.; CARVALHO, E. J. M.; CORREAA, P. C. S. Matéria orgânica e atributos físicos de argilosso amarelo distrófico no Nordeste paraense. Amazônia: Ciência e Desenvolvimento, v.5, n.9, p.187-198, 2009.

VIEIRA, L. S.; SANTOS, P. C. T. C. dos. Amazônia: seus solos e outros recursos naturais. São Paulo: Ceres (Agricultural Research Communication Centre), 1987. 